\title{
Ranking of alternatives for emergency routing on urban road networks
}

\author{
M. Woelki, T. Lu \& S. Ruppe \\ Traffic Management, German Aerospace Center, \\ Institute of Transportation Systems, Germany
}

\begin{abstract}
Routing on urban road networks for emergency cars is an application of Dijkstra's algorithm with relevance in everyday-life. Since distances in urban transport are rather short it is computationally possible to calculate many paths and compare them afterwards. This paper uses Dijkstra's k-shortest path algorithm in order to calculate shortest and fastest paths and finally finding an ordering of alternatives for multi-criteria routing. The solutions are displayed in criterion space and the Pareto front is identified. Routes are ranked according to the normalized weighted-sum method. Obviously, the more alternatives there are the more possibilities for the emergency car to circumscribe traffic jams. Therefore 'close alternative routes' are taken into accounts that share a certain fraction of nodes with one Pareto optimal route. To those bundles of routes a ranking is assigned that may serve as recommended action for the driver.

Keywords: routing, Dijkstra's algorithm, weighted-sum method.
\end{abstract}

\section{Introduction}

In contrast to 'dynamic routing' of modern navigation systems where the current position of the traveller during his journey is continuously fed back to calculate a current best route, in 'static routing' a route between origin and destination (O/D relation) on a transportation network is calculated once, typically before the trip.

Usually those routings are performed for private cars but also routing strategies for emergency cars exist (Woelki et al. [1]). Although the results of the present paper are easily generalizable to private transport, here the focus is on routing for emergency cars. One example of a platform providing static routing is Google Maps (Google [2]), a very famous route planning software. The cores 
of all those systems are so-called shortest path algorithms (Delling et al. [3]) that have a long tradition since Dijkstra's initial work (Dijkstra [4]) in 1959. His famous paper shows how the optimal path for a given O/D relation can be found with regard to a certain weight function (typically the length) associated with the edges of the graph. Google Maps provides for a given O/D relation up to three different 'reasonable' routings which are 'substantially different' (Abraham [5]). However, which route is the best in practice cannot be stated generally since it depends on different factors, such as length of the route, travel time, cost, personal preferences of street types, etc. The theory of optimizing such a variety of quantities is known as multi-objective optimization (Martins [6)].

In the context of optimization, so-called Pareto-optima are of special importance. If one considers the case of two criteria, those are routes for which one cannot find a second route in which the one quantity is smaller and the second quantity is the same or smaller. Each Pareto-optimal route may dominate other routes. This is the case for all routes in which both quantities are not smaller than the two quantities in the corresponding Pareto optimum. For recent investigations on multi-criteria Pareto search, see Müller-Hannemann and Schnee [7]. The authors of Climaco and Martins [8] attacked the problem in the following way: In order to find the optimal routes with regard to two quantities that shall be minimised, find the k-shortest paths for the first quantity until the route appears in which the second quantity takes its minimal value. Then, from those k paths, identify the Pareto-optimal routes. This is mainly the technique applied here. Surely, in a bi-criteria minimization problem, a Pareto optimum is a priori better than all the routes that it dominates. However it might be that a dominated route appears to be better than another Pareto optimal route. Therefore the following paper considers the whole set of routes and tries to find a performance order (ranking) of those routes.

\section{Problem definition and overview}

The problem addressed in this paper is the following: An emergency car should take an optimal route from its fire station to a destination. In Germany, emergency cars have to reach their destination within a legal time of 8 minutes. Therefore, in the following one special route is considered in which the travel time is close to this limit of 480 seconds. In calculating the travel times on each edge (street section) it is assumed that the velocity equals the speed limit. Therefore, in reality the travel times for the various routes may differ from those of the present paper. The driver should try to minimize the risk of unforeseen events that prevent him from reaching the destination within the legal time. The idea is that the longer the path is, the higher the probability for unforeseen events and the higher becomes the risk. Hence, it should be tried to minimize path length and travel time at the same time. Section 3 presents simulation results for minimization of either travel time or path length. Section 4 considers bi-criteria optimization methods that give an optimal route with regard to both, travel time and path length, and presents a ranking of alternatives. So, if there is some reason for the fireman not the take the optimal route, he can choose from a 
selection of alternative routes. The worst that can happen is that the emergency car is blocked for example in traffic jam and there is no option for a rescue alley. Therefore, routes are desirable that have close alternative routes that share a number of nodes with the original route, so that obstacles can be surrounded spontaneously. Close alternatives should itself be efficient with regard to travel time and path length which gives rise to a ranking of whole bundles of routes, see Section 5.

\section{Routing results}

Figure 1 shows the scenario considered in this paper: A routing from Feuerwehrstraße 1 in Brunswick, the location of the fire station from where the emergency cars start, to a destination in Weststadt. Displayed are only the Pareto optimal routes. Red: 'shortest' (Pareto \#1), yellow points: Pareto \#2. Orange: 'fastest' (Pareto \#4), Blue and cyan points correspond to a Pareto optimum (Pareto \#3) appearing in both sets. The screenshot is taken from Google Earth [9]. The paths are obtained from k-shortest path routing and k-fastest path routing, as is presented in the following.

\subsection{The k-shortest path and k-fastest path routing}

The set of paths is composed of the 1000 shortest and the 1000 fastest paths ( $\mathrm{k}=1000$ in both cases). Both routings complement well one another since it turns out, that there is only a small overlap of 110 routes among the best 1000 paths in each variant.

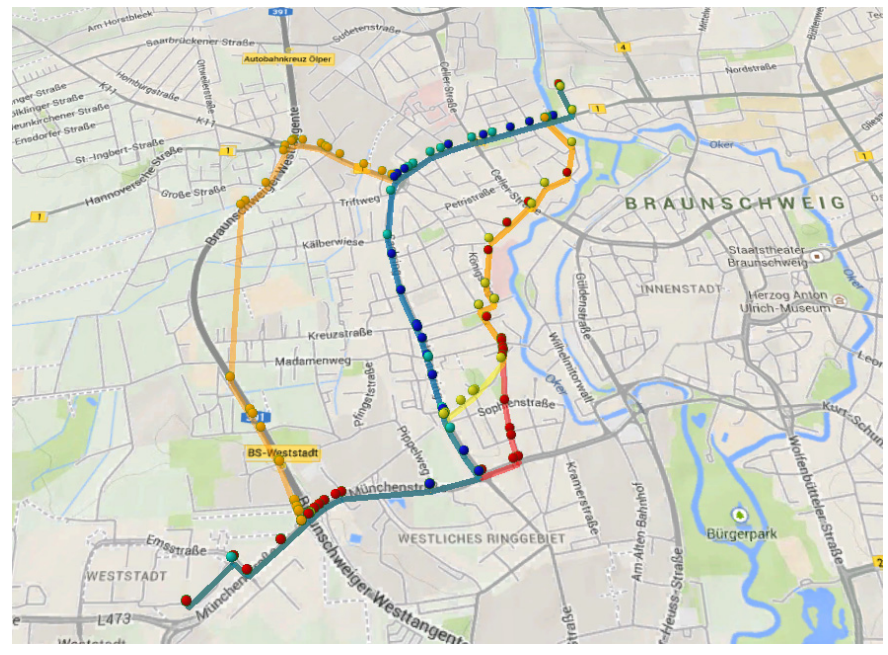

Figure 1: The four different Pareto optimal routes. 
Figure 2 shows the 1890 solutions in criterion space. The four Pareto optima are visualized as squares in the colour according to Figure 1. Further the dominated paths are depicted with colouring according to their weight. The fastest route (orange square) has coordinates $(304 \mathrm{~s}, 5019 \mathrm{~m})$. The shortest path corresponds to $(432 \mathrm{~s}, 4497 \mathrm{~m})$. This Pareto optimum is depicted as the red square. The points representing the 1000 fastest paths are limited to the right by a travel time of around 370 seconds, corresponding to the slowest of those paths with $(368 \mathrm{~s}, 5920 \mathrm{~m})$. Accordingly, the points from shortest path routing are limited from above by around 4900 meters, corresponding to the furthest of the 1000 shortest paths with coordinates $(460 \mathrm{~s}, 4918 \mathrm{~m})$. Therefore, there is a rectangular region that contains no solutions. The Pareto optimum at (332s, $4606 \mathrm{~m}$ ), depicted as a blue square is covered by both, fastest and shortest path routing. It is at the same time the $48^{\text {th }}$ fastest route and the $12^{\text {th }}$ shortest route.

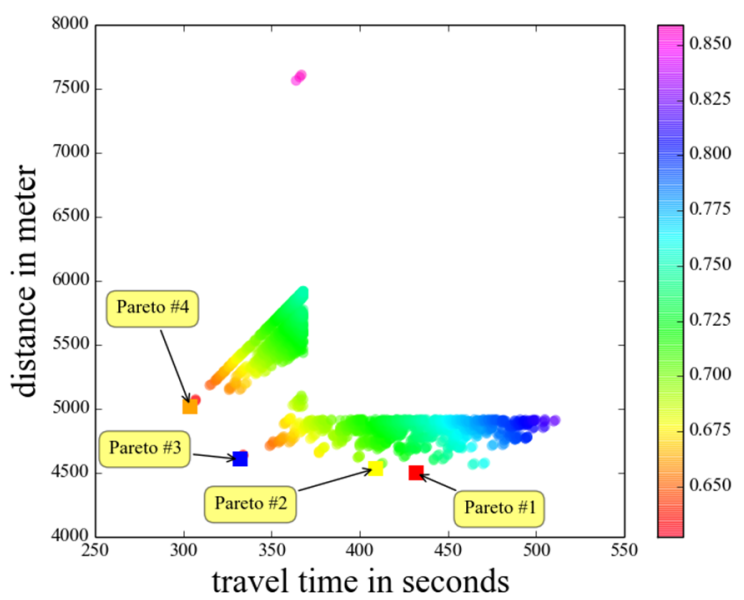

Figure 2: $\quad$ Route results in criterion space.

\section{Bi-criteria optimization}

Having a set of routes at hand, along with their travel times and path lengths, one can decide which one is optimal. Further, a natural ranking from best to worst is obtained. As initial set of paths, the routes among the k-shortest and k-fastest paths are considered. A classic example of multi-criteria optimization is the normalized weighted sum method (Marler and Arora [10]) which is applied to the routing problem in the following. With each of the routes $r$ one associates a total weight

$$
W_{r}=0.5 \cdot \frac{L_{r}-L_{\min }}{L_{\max }-L_{\min }}+0.5 \cdot \frac{T_{r}-T_{\min }}{T_{\max }-T_{\min }} .
$$

Here, $L_{\min }$ is the length of the shortest path, $L_{\max }$ the length of the k-shortest path (in this paper, this is the $1000^{\text {th }}$ shortest path) and $T_{\min }$ is the travel time of the fastest path, while $T_{\max }$ is the travel time of the k-fastest path. 
This choice takes path length and travel time equally into account. The values of $W_{r}$ (for $r=1,2, \ldots, k$ ) give a natural ranking of alternatives with $W_{l}$ representing the optimal path.

In Figure 2 the four Pareto optima are visualized as squares in the colour according to Figure 1. Further, the dominated paths are depicted with colouring according to their weight.

Table 1 shows the rankings of the four Pareto optima. The first column labels the routes according to their length. One sees that the weighted sum method favours the fastest path $(304 \mathrm{~s}, 5019 \mathrm{~m}$. The other two Pareto optima are ranked $304^{\text {th }}$ and $755^{\text {th }}$ and thus are included in the $16 \%$ to $40 \%$ of favoured paths.

Table 1: Results for the four Pareto optima.

\begin{tabular}{|c|c|c|c|}
\hline Pareto \# & Travel time/s & Path length/m & Ranking \\
\hline 1 & 432 & 4497 & 755 \\
\hline 2 & 409 & 4533 & 304 \\
\hline 3 & 332 & 4606 & 2 \\
\hline 4 & 304 & 5019 & 1 \\
\hline
\end{tabular}

\section{Classification of routes}

In this section, the four Pareto routes are considered as the basic route options and all the other routes will be brought in relation to those Pareto routes. Obviously one could also choose other routes that serve as strategic alternatives: Completely disjoint routes for instance that only agree in start and target nodes and that correspond to different strategic options as motorway, Main Street, etc. However, for simplicity and for the sake of generality it is stuck to the Pareto optima here.

The concept used to identify a bundle of close alternatives is the following: A route 'belongs to' the set of close alternatives to Pareto route $\# n$, if the percentage of shared nodes with Pareto route $\# \mathrm{n}$ is higher than the percentages of shared nodes with the other Pareto routes.

The four diagrams of Figure 3 show for each of the four Pareto optima all routes that belong to the respective Pareto route. Top left: 406 routes belonging to Pareto route \#1 (21\% of all routes), top right: 367 routes belonging to Pareto route \#2 (19\%), bottom left: 245 routes belonging to Pareto route \#3 (13\%), bottom right: 872 routes belonging to Pareto route \#4 (46\%). One sees that the Pareto routes \#1 to \#3 are located in the same region of the criterion space and that the fastest route (Pareto route \#4) is located in a different region. This is a consequence of the fact, that route \#4 is maximally disjointed from the others, especially from route \#1 (see Table 2).

For the Pareto optima \#3 and \#4 Figure 3 shows that they dominate all routes that belong to them (and those paths have both larger travel time and larger path 
length which is typically associated with small detours from the main route). However for Pareto optima \#1 and \#2 (which agree by around $80 \%$, see Table 2) there are also paths that they do not dominate (those are the paths 'to the left' of Pareto \#1 and Pareto \#2). In fact those routes are dominated by Pareto \#3. They obviously have larger path lengths but smaller travel times.

Table 2: Percentage of the number of common nodes between two Pareto routes.

\begin{tabular}{|c|c|c|c|c|}
\hline Pareto \# & $\mathbf{1}$ & $\mathbf{2}$ & $\mathbf{3}$ & $\mathbf{4}$ \\
\hline $\mathbf{1}$ & $100 \%$ & $86 \%$ & $47 \%$ & $26 \%$ \\
\hline $\mathbf{2}$ & $82 \%$ & $100 \%$ & $51 \%$ & $24 \%$ \\
\hline $\mathbf{3}$ & $41 \%$ & $47 \%$ & $100 \%$ & $49 \%$ \\
\hline $\mathbf{4}$ & $22 \%$ & $22 \%$ & $49 \%$ & $100 \%$ \\
\hline
\end{tabular}
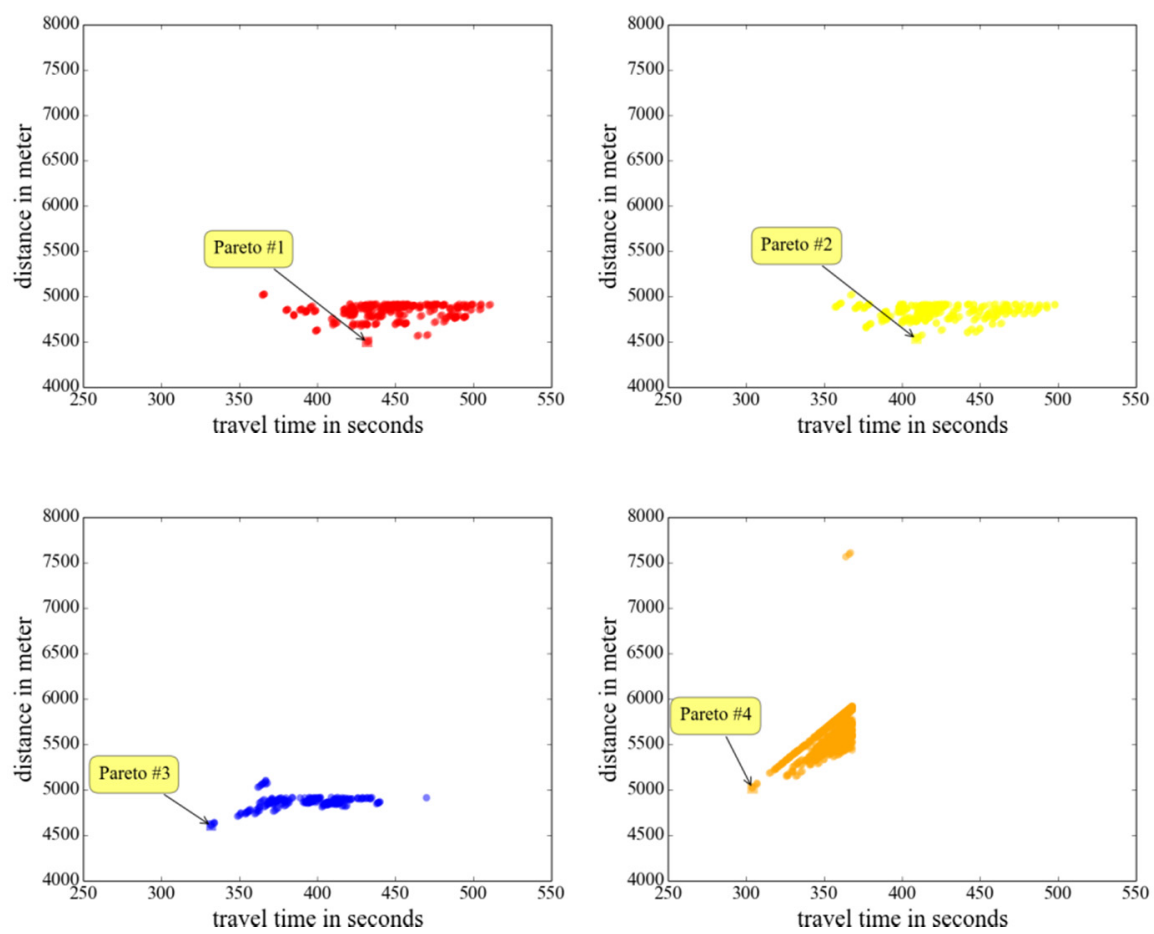

Figure 3: Routes that belong to one of the four Pareto routes.

So, an explanation would be that those routes make a detour over the faster Pareto route \#3 (the part of this route that is not covered by Pareto routes \#1 and \#2, see Figure 1) but share more nodes with Pareto route \#1 or \#2. 
In order to decide for given number of alternatives $a_{p}$ and average weight $w_{p}$ which bundle of routes belonging to Pareto optimum $p$ is better than another, the normalized weighted sum method is applied again. The formula for weights that naturally define an increasing ranking is rewritten as

$$
G_{p}=0.5 \cdot \frac{-a_{p}-872}{-245+872}+0.5 \cdot \frac{w_{p}-0.7052}{0.7557-0.7052} .
$$

Note that maximizing the number of close alternatives is equivalent to minimizing its negative. Results are shown in Table 3.

Table 3: Comparison of bundles of close alternatives and generalized ranking.

\begin{tabular}{|c|c|c|c|c|}
\hline Pareto \# & \#close alternatives & av. weight & gen. weight & gen. ranking \\
\hline 1 & 406 & 0.7557 & 0.872 & 4 \\
\hline 2 & 367 & 0.7328 & 0.676 & 3 \\
\hline 3 & 245 & 0.7052 & 0.5 & 2 \\
\hline 4 & 872 & 0.7160 & 0.108 & 1 \\
\hline
\end{tabular}

One sees that for the present O/D relation the ranking is in line with the ranking given in Table 1. The fastest path Pareto \#4 (the orange route in Figure 1 ) is favoured, since it has the smallest generalized weight $G_{4}$. Second favourite is Pareto \#3 (the blue route in Figure 1) and so on.

\section{Summary and outlook}

A strategy for static routing of emergency cars on an urban road network was presented. It was argued that it is desirable to minimize both, travel time and path length simultaneously. The argument for minimizing path length as well is that larger paths have higher probability for unforeseen events especially traffic jams or other obstacles for the emergency car. To this aim, many different routes have been obtained to which the normalized weighted sum method was applied and a ranking of routes with regard to their desirability has been obtained. In order for the emergency car to be able to circumscribe obstacles it has been pointed out that desirable routes have many close alternative routes. Since those routes each have a weight that shall be minimized, one arrives at another bicriteria optimization problem. This has been solved once again with the normalized weighted sum method. The final result of this paper is a ranking of bundles of routes according to their desirability considering their average weight with respect to travel time and path length and the amount of alternatives. This ranking serves as a recommended action for the driver.

While this paper made some technical simplifications, future work could generalize upon the present work. For example, one could exclude loops from the k-shortest path routing to discard absurd routes. Further it was assumed that 
emergency cars drive always exactly at the speed limit, here it would be interesting to take realistic velocities into account.

For future research it would be interesting to generalize the results of the present paper to the case of multiple objectives. In Woelki et al. [1] also the number of lanes was considered. This could be included in the normalized weighted sum method as well. Furthermore in [1] a number of criteria are mentioned that have relevance to emergency routing, for example if there are guarded level crossings. Obviously all those routes in which the emergency car has to pass a guarded railway crossing have to be deleted from the set of routes.

\section{References}

[1] Woelki M., Nippold R., Bonert M., and Ruppe S. Risk-minimal routes for emergency cars. In: Disaster Management and human health risk III, 159169. WIT Press. Disaster Management and human health risk III, 09.-11. July 2013, a Coruna, Spain. ISBN 978-1-84564-738-4. ISSN 1746-4498 (2013)

[2] Google maps.google.com

[3] Delling D., Sanders P., Schultes D., and Wagner D., Engineering Route Planning Algorithms, in Lerner J., Wagner D., and Zweig K. A. (Eds.): Algorithmics, LNCS 5515, pp. 117-139, Springer-Verlag Berlin Heidelberg (2009)

[4] Dijkstra E.: A note on two problems in connection with graphs, Numer. Math. 1 pp. 269-271 (1959)

[5] Abraham I., Delling D., Goldberg A. V., and Werneck R. F.: Alternative Routes in Road Networks. Experimental Algorithms Lecture Notes in Computer Science Volume 6049, pp. 23-34 (2010)

[6] Martins E. Q. V., On a Multicriteria Shortest Path Problem, European Journal of Operational Research, 26(3): pp. 236-245 (1984)

[7] Müller-Hannemann M., Schnee M.: Finding All Attractive Train Connections by Multi-criteria Pareto Search. In: Geraets F., Kroon L. G., Schoebel A., Wagner D., Zaroliagis C. D. (eds.) Railway Optimization 2004. LNCS, vol. 4359, pp. 67-90. Springer, Heidelberg (2007)

[8] Climaco J. C. N. and Martins E. Q. V.: A bicriterion shortest path algorithm, European Journal of Operational Research 11 pp. 399-404 (1982)

[9] earth.google.de, 2014-04-03

[10] Marler R. T., Arora J. S.: The weighted sum method for multi-objective optimization: new insights, Struct. Multidisc. Optim. 41: pp. 853-862 (2010) 